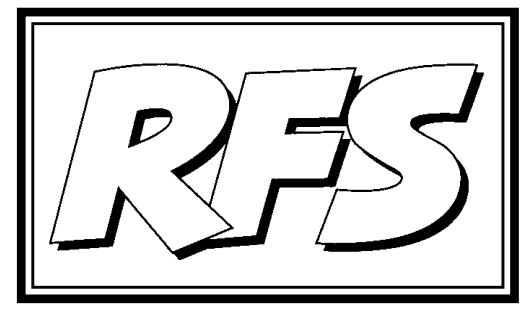

Revista de Fomento Social, 61 (2006), 213-235

\title{
Balance de las relaciones económicas de México con la Unión Europea. ¿Confianza en el regionalismo Norte-Sur?
}

(Palabras clave: Relaciones económicas, América Latina, MÉxico, UE, Regionalismo, InTEGRACIÓN REGIONAL, Comercio, CoOperación, DesarRollo.

KEY WORDS: ECONOMIC RELATIONS, LATIN AMERICA, MEXICO, EU, REGIONALISM, REGIONAL InTEGRATION, TRADE, COMMERCE, COOPERATION, DEVELOPMENT)

1 Profesor del Tecnológico de Monterrey (ITESM-CEM), México. El autor agradece la colaboración de Maxaira Baltazar, Marisol Fondado y Dulce Lupián. Agradece también los comentarios y sugerencias del Consejo de Redacción de la Revista de Fomento Social y del evaluador anónimo. Sin embargo, las conclusiones y posibles errores son responsabilidad exclusiva del autor. 


\section{Introducción}

Desde mediados de los años 80 , y como reacción a la crisis de la deuda, los países latinoamericanos empezaron a aplicar políticas económicas de corte ortodoxo, de acuerdo a la propuesta de los Programas de Estabilización y de Ajuste Estructural (PAE) del Fondo Monetario Internacional (FMI) y del Banco Mundial (BM), cuyos principios básicos se conocen como 'consenso de Washington'. Paralelamente, surgió la Empresa para la Iniciativa de las Américas (Enterprise for the Americas Initiative) de 1990, que derivaría hacia la propuesta del Área de Libre Comercio para las Américas (ALCA) hecha a partir de la Cumbre de las Américas de Miami en diciembre de 1994; el Tratado de Libre Comercio de Norteamérica entre Canadá, Estados Unidos y México (TLCAN) ${ }^{2}$ de 1994; el Acuerdo de Asociación Económica, Concertación Política y Cooperación entre la Unión Europea y México (TLCUEM) ${ }^{3}$ que entró en vigor en el año 2000.

Estos acuerdos se producían al mismo tiempo que el regionalismo latinoamericano se renovaba respecto a los años anteriores y en el que la integración regional entre los países de la región ${ }^{4}$, de acuerdo con el modelo europeo y estructuralista (renano), se veía en transformación y en convivencia con las nuevas propuestas regionales provenientes del mundo anglonorteamericano (sajón) con el referente del TLCAN.

La nueva lógica de construcción regional en América Latina ha tenido como elementos básicos: contribuir a la liberalización del comercio mundial

2 En inglés North American Free Trade Agreement (NAFTA).

3 De acuerdo con las siglas, Tratado de Libre Comercio entre la Unión Europea y México (TLCUEM) es la forma en que se conoce al Acuerdo de Asociación Económica, Concertación Política y Cooperación entre la Unión Europea y México. Al nominar de esta manera a este Acuerdo se le da un carácter predominante a la relación comercial, pasando a un segundo plano otros aspectos de éste. Sin embargo, el libre comercio entre la Unión Europea y México forma parte de la Asociación Económica, y ésta última es uno de los tres pilares del Acuerdo, junto con la Concertación Política y la Cooperación. El acuerdo de cuarta generación entre La UE y México fue el primer acuerdo de este tipo que entró en funcionamiento en el marco de la relación birregional UE y América Latina. El 1 de julio de 2000 entró en vigor y desde entonces ha supuesto un desarme progresivo (normas comerciales, de competencia, mercados públicos y servicios) que culminará en el año 2007.

4 Aplicaremos el término "región" cuando nos estemos refiriendo a Latinoamérica en conjunto y los términos "acuerdos regionales" o "regionalismo" cuando nos estemos centrando en agrupamientos más pequeños de países latinoamericanos entre ellos o con otros países o bloques que no forman parte de la "región". 
a través de la apertura unilateral o de la profusión de acuerdos comerciales y de integración con otros países o bloques, y atraer hacia ellos la Inversión Extranjera Directa (IED). Estas iniciativas regionales han sido paralelas a la aplicación de la política económica de la nueva ortodoxia regional derivada de los PAE, que han consistido básicamente en la búsqueda del equilibrio macroeconómico a través del control de la inflación; del estímulo a la inversión privada; de un mejor acceso a los recursos financieros, con el afán de lograr precios internacionalmente competitivos; $y$ de un crecimiento estable y duradero. Y todo ello en un amplio, más eficiente, competitivo y creciente espacio económico, capaz de competir mundialmente.

En este momento, y con el TLCAN como ejemplo paradigmático, aparecieron los Acuerdos Regionales Norte-Sur ${ }^{5}$ junto a los tradicionales Acuerdos Regionales Norte-Norte y Sur-Sur. Los países de menor desarrollo que se fueron incorporando a este tipo de acuerdos esperaban que éstos les permitieran alcanzar los niveles de desarrollo económico y social del mundo desarrollado. En toda la región, el TLCAN, el ALCA y sus recientes transformaciones en tratados de libre comercio bilaterales (TLC); y los acuerdos de última generación, como el TLCUEM, donde la consolidación de una zona de libre comercio, en el marco de la concertación económica, se convierte en un pilar fundamental junto con los pilares de concertación política y cooperación. Estos acuerdos han vuelto a poner en primer plano en América Latina, y en México, la discusión sobre los beneficios y riesgos de las distintas formas de agrupamiento regional y su naturaleza teórica. No cabe duda que tanto el regionalismo impulsado desde Estados Unidos y desde la UE tiene un papel referencial en América Latina, al igual que la naturaleza, metas y objetivos de sus relaciones políticas y económicas con los países o bloques regionales latinoamericanos. Este hito en la historia económica regional inauguró una nueva era para el regionalismo que se convirtió, a partir de ese momento, en un fenómeno más complejo.

Tras más de una década de aplicación de estas políticas económicas y la aparición en escena de lo que se ha llamado en América Latina nuevo regionalismo (ver Devlin y Estevadeordal [2001]) -de los que tanto México como Argentina se erigieron, de distinta forma y por diversos motivos en sus

5 El regionalismo Norte-Sur es aquel en el que los países de altos ingresos y los países en desarrollo actúan en pie de igualdad en un acuerdo regional. Hasta los años noventa los acuerdos regionales en el mundo, eran esencialmente Norte-Norte (entre países de altos ingresos) y Sur-Sur (entre países en desarrollo) (ver Schiff y Winters [2004]). 
"buques insignia"-, se ha vuelto a abrir el debate sobre la conveniencia de su aplicación para que los países latinoamericanos alcancen una adecuada inserción internacional y generen los cambios estructurales necesarios que logren mejorar los niveles de vida para sus ciudadanos. Sin duda, el TLCAN ha supuesto uno de los hitos más importantes en el regionalismo contemporáneo latinoamericano porque, a partir de su firma y puesta en funcionamiento, como se ha mencionado, se empezó a transformar la esencia de los acuerdos regionales entre los países de la región con países de fuera de ella. Esencia a la que no ha sido ajeno el nuevo modelo de relación de la UE con México y con el resto de los países del subcontinente.

Dentro de este marco, en los últimos años de la década del noventa y a principios de la presente, Argentina sufrió una profunda crisis económica, política y social que acabó con el esquema de la convertibilidad y con la imagen de este país como alumno aventajado del modelo de desarrollo neoliberal diseñado desde Washington. Decía Paul Krugman en un artículo en el año 2002 en relación a la situación argentina:

\begin{abstract}
"Aunque las imágenes de los disturbios en Argentina han pasado fugazmente por las pantallas de nuestros televisores, apenas le importa a nadie en Estados Unidos. No es más que otro desastre en un país pequeño y lejano, del que no sabemos nada, un país tan remoto como, por ejemplo, Afganistán, y con las mismas remotas posibilidades de afectar a nuestras vidas (...) no hago esta comparación a la ligera. Puede que la mayoría de la gente de aquí crea que esto no es más que otra terrible crisis latinoamericana, pero para gran parte del mundo las políticas económicas de Argentina tenían estampada por todas partes la etiqueta made in Washington (...) Se redujeron aranceles, se privatizaron empresas públicas, se abrieron las puertas a las multinacionales y el peso se fijó al dólar (...) El pasado abril, George W. Bush volvió a vender la propuesta del Área de Libre Comercio de las Américas como un importante objetivo de política exterior, que 'construiría una era de prosperidad en un hemisferio de libertad'. No lloren por Argentina, lloren con ella" (Krugman [2002]).
\end{abstract}

Esta reflexión de Krugman también es en gran medida aplicable a México, puesto que este país fue el primero en desarrollo en firmar un acuerdo de libre comercio con países desarrollados (Estados Unidos y Canadá) y posteriormente, el primero que firmó un acuerdo de cuarta generación con la UE.

Este desarrollo del regionalismo actual en toda América Latina convierte a México, y al TLCAN como modelo regional dentro del engranaje de las políticas ortodoxas, en un referente para el resto de los países de la región de poderoso valor simbólico. Parafraseando a Krugman, si el TLCAN es valorado positivamente-tras más de 10 años de su entrada en vigor-como parte de la estrategia de desarrollo para México, ésta será un motivo de alegría para México, para todos los países de América Latina y por supuesto para 
Estados Unidos como potencia hegemónica en este tipo de acuerdos; pero si la valoración es de signo contrario, con más sombras que luces, todos los países mencionados se deberán sentir preocupados por y con México.

Al mismo tiempo, es importante preguntarse por el modelo regional que la UE está impulsando en América Latina, donde México también se ha erigido en referente. La valoración del TLCUEM tras sus primeros años de aplicación también despierta interrogantes en la región y en México. Si los acuerdos con la UE no se tornan en esencia diferentes a lo acuerdos impulsados desde Estados Unidos, éstos no estarán ofreciendo un camino alternativo para las relaciones Norte-Sur y por lo tanto se verán arrastrados por el efecto y las valoraciones hechas al TLCAN. Hecho que mantedría a la UE como un actor secundario en la región y cerraría a los países latinoamericanos algunas encrucijadas regionales.

Este trabajo reflexiona sobre el nuevo regionalismo en el que está participando México, especialmente el que está tenido lugar con la Unión Europea, y su poder referencial sobre el resto de los países y agrupamientos regionales en América Latina. México puso en marcha estos acuerdos asimilándolos a una estrategia de desarrollo en sí misma. Sin embargo, los resultados sobre el desarrollo mexicano, de la participación en estos acuerdos, han sido decepcionantes. En un momento, en que varios países y procesos de integración de la región se encuentran negociando y firmando acuerdos con Estados Unidos y La Unión Europea, la valoración del caso mexicano, y del carácter distintivo del TLCUEM, se vuelve especialmente trascendente dentro del debate actual en América Latina sobre la relación entre comercio, cooperación y desarrollo.

\section{Las relaciones regionales e internacionales de América Latina bajo el espejo europeo}

Las relaciones actuales de la UE con México, y con el resto de los países de América Latina, no han sido ajenas a la influencia del TLCAN. Sin embargo, tampoco conviene olvidar que entre América Latina y Europa existen cinco siglos de historia compartida, en los que se ha dado una relación de ida y vuelta entre ambos. Como señala Patricia Galeana: "además de la América descubierta e inventada por Europa, existe una Europa descubierta e inventada por América". El historiador inglés John Hale señala: "Gracias a la suprema confianza que les inspiró el descubrimiento de América, los euro- 
peos hicieron un mayor esfuerzo por plantearse la cuestión de su identidad, sobre todo cuando la simple curiosidad por los hombres y las costumbres de los demás continentes iba acompañada de comparaciones -no siempre tranquilizadoras-, entre europeos y americanos" (Galeana [1999] p. 93). Normalmente se le ha dado un mayor énfasis al referente europeo en América Latina, aunque la relación en la dirección contraria, como menciona Hale, no ha sido menos importante. Hoy esta doble mirada, en nuestra opinión, sigue siendo importante en un momento en que una parte de Europa se plantea cómo afrontar los retos de su construcción regional (la integración de los países de la Europa del Este, su integración política y su papel como bloque en el contexto internacional), y cuando los esquemas de integración o los acuerdos preferenciales de comercio y cooperación son una prioridad urgente y presente para América Latina. "La intensa dinámica del cambio en que estamos inmersos Europa y América Latina exige un esfuerzo de información, análisis y evaluación de la coyuntura actual para superar los vacíos de identidad propios, de crisis históricas del inicio de una nueva era. Ante la llegada del nuevo milenio, debemos replantear nuestras relaciones en un esquema de respeto y crecimiento mutuo" (Ibídem p. 104).

Muchos de los procesos de integración en América Latina han puesto su mirada en la integración europea y han proyectado sus construcciones regionales con el proyecto europeo en su horizonte. A principios del siglo XXI, la UE está explorando distintas alternativas de avance en su proceso de integración y los procesos regionales en América Latina, bajo el nuevo marco planteado desde los Estados Unidos a través de los TLCs, conviven con los dos modelos regionales referenciales: el tradicional de inspiración europea-estructiralista ${ }^{6}$ y el anglonorteamericano ${ }^{7}$ (ver Casas [2005]).

Las relaciones entre Europa y América Latina, donde se incluye México, a partir de la Segunda Guerra Mundial se dividen en cuatro etapas que se

6 Es un modelo de regionalización de naturaleza intergubernamental y supranacional; con objetivos de integración económica, política y social; y abarcan temas económicos (desde el intercambio libre de bienes servicios, y de los llamados 'nuevos temas' entre los países miembros, hasta la constitución de una unión económica y monetaria), políticos (por ejemplo, democracia y proyectos de unidad política), y sociales (por ejemplo, circulación de personas, educación, etc.).

7 Es un modelo exclusivamente intergubernamental; con el objetivo fundamental de lograr la integración comercial (intercambio libre de bienes, servicios, y los 'nuevos temas' entre los países miembros) y de cooperación en asuntos puntuales (por ejemplo: fomento de la democracia). 
corresponden con las características de los acuerdos comerciales y de cooperación que se han firmado entre ambas regiones.

Los acuerdos de primera generación aparecen a principios de los setenta con el objetivo, por parte de Europa, de expandir los intercambios comerciales. En esta época se creó el Sistema Generalizado de Preferencias (SGP). Éste consistió en el trato preferencial concedido por Europa a productos originarios de países en desarrollo de conformidad con el Sistema Generalizado de Preferencias del GATT. En esta década con México se establecieron los primeros mecanismos encaminados a "ampliar la cooperación comercial y económica" entre ambos agentes internacionales, dando lugar al surgimiento de la Comisión Mixta, la cual a su vez promovería el primer Acuerdo de Cooperación entre México y la actual UE en 1974.

Los acuerdos de segunda generación datan de comienzos de los años ochenta y tenían el objetivo de ofrecer un apoyo político para la pacificación de Centroamérica y la democratización en el resto de los países latinoamericanos. En 1984, España y Portugal -todavía no eran miembros de la actual UE- participaron en un encuentro con el Grupo Contadora para llevar adelante una iniciativa de paz para Centroamérica. El Grupo Contadora se amplió con el apoyo de los países sudamericanos y se convirtió en 1986 en el Grupo de Río ${ }^{8}$. A partir de 1987, se creó un marco de diálogo a nivel de ministros de Relaciones Exteriores entre el Grupo de Río y la actual UE, que se institucionalizó en la Declaración de Roma del 20 de diciembre de 1990. Desde entonces se celebran anualmente reuniones ministeriales institucionalizadas entre ellos, exceptuando los años en que tienen lugar las Cumbres de Jefes de Estado de la Unión Europea y de América Latina.

En los años noventa, la UE le dio un mayor peso a la cooperación al desarrollo, acuerdos de tercera generación, basados en valores globales, la democracia, los derechos humanos y el desarrollo sostenible. Estos son los años que ven como el Acuerdo entre México y la UE de 1974, que había otorgado al país latinoamericano el reconocimiento como país en desarrollo

8 Surgido en 1986, de la convergencia entre el Grupo Contadora, formado por Colombia, México, Panamá y Venezuela y el denominado Grupo de Apoyo, integrado por Argentina, Brasil, Perú y Uruguay, el Grupo de Río es hoy el mecanismo por excelencia de consulta y concertación política regional. El Grupo cuenta, actualmente, con la participación de 18 Estados de Latinoamérica, incluyendo los países centroamericanos y la República Dominicana, recientemente admitidos como miembros plenos, y un representante rotativo de los países del Caribe (Forero [1999] p. 202). 
y ciertas concesiones en materia comercial, se transforma en el Acuerdo Marco de Cooperación de 1991 (Cruz [2003] pp. 160-161) que en sintonía con el diseño de las relaciones birregionales amplía la cooperación económica y comercial, a otros ámbitos como el tecnológico, agrícola, de medio ambiente, comunicaciones, entre otros.

A partir de 1994, y motivados entre otros acontecimientos en ambas regiones por la firma del TLCAN, se empiezan a negociar los acuerdos de cuarta generación entre la UE y MERCOSUR, Chile y México. Estos acuerdos buscan el fortalecimiento de las relaciones entre las partes basándose en los principios de reciprocidad y de intereses comunes, estableciendo un modelo de asociación política y económica y cuyo objetivo a mediano plazo es el establecimiento de zonas de libre comercio que incluyan una mayor cooperación política, científico técnica, social y cultural, además del ámbito comercial (donde se contemplan, básicamente, los mismos temas que en el TLCAN).

Sin embargo, la consolidación del TLCUEM, durante la negociación, implicó ciertos retos para ambas administraciones, no sólo por la amplitud de la unión planteada y la variedad de intereses nacionales al interior de la misma, sino también por la trascendencia que el mismo representaba para México en materia doméstica, a través de la llamada "cláusula política", la cual estaba enmarcada en un contexto nacional donde en los últimos setenta años había gobernado un solo partido. Sin duda, la negociación del TLCUEM en la segunda mitad de la década de los noventa tuvo un efecto político en México, que favoreció: la firma del Acuerdo San Andrés Larrainza en 1996 entre el Gobierno Mexicano y el Ejercito Zapatista de Liberación Nacional (EZLN) que permitió relajar la tensión y violencia en Chiapas -una de las zonas con un mayor número de población indígena y con elevados niveles de pobreza-; y la alternacia democrática en el año 2000 y el comienzo de "la transición democrática" en este país. Asimismo, hubo discrepancias a lo largo de las rondas de negociación entre la UE y México respecto a cuestiones referentes al acceso a mercados y reglas de origen que frenaron ocasionalmente la consolidación de los lazos de cooperación.

Hasta el momento, los acuerdos de cuarta generación entre la UE y América Latina que se han puesto en marcha son los acuerdos con Chile y con México. Además, en la Cumbre Eurolatinoamericana de Viena (2006) se tomó la decisión de entablar negociaciones relativas al Acuerdo de Asociación entre la UE y Centroamérica, y de continuar indigando la posibilidad de comen- 
zar las negociaciones del Acuerdo con la subregión andina. Sin embargo, el proceso andino de integración vive una grave crisis interna detonada por la salida de Venezuela y por cierta incertidumbre respecto a la futura participación de Bolivia. Todos estos elementos, junto con los escasos avances en la integración económica de la subregión andina, están haciendo que la UE esté posponiendo el inicio de la negociación de un acuerdo de asociación estratégica y libre comercio entre ambos bloques hasta que en la integración andina se den las condiciones necesarias para ésto.

En general, los dirigentes latinoamericanos perciben que existe una estrecha relación entre la activación de los acuerdos de cuarta generación con la UE y las negociaciones de los países o subregiones latinoamericanas con Estados Unidos en el marco de acuerdos de libre comercio tipo Tratados de Libre Comercio (TLC) o el Acuerdo de Libre Comercio para las Américas (ALCA). De ahí la conocida frase que circula entre los funcionarios internacionales latinoamericanos "de que si quieres negociar con Bruselas vete a Washington”. La reciente firma del Tratado de Libre Comercio entre República Dominicana, Centroamerica y Estados Unidos (DR-CAFTA) ${ }^{9}$, del Tratado de Libre Comercio con Colombia, y el avance en las negociaciones para las firmas de este tipo de tratados o acuerdos con Perú, podrían generar el efecto rebote que acelerara en los próximos meses las negociaciones con el proceso de integración centroamericano y que hiciera que la UE finalmente lanzara las negociaciones para la firma de un acuerdo de libre comercio con la subregión andina conjuntamente o, de manera bilateral, con Colombia y Perú..

\section{3 . Evolución de las relaciones comerciales en el marco del acuerdo de asociación entre México y la Unión Europea ${ }^{10}$}

Después de la firma de diversos tratados internacionales, entre ellos el TLCAN y el TLCUEM, México ha aumentado su comercio total a poco más de 435 mil millones de dólares en 2005, lo que representa un aumento de

9 En inglés Dominican Republic, Central American Free Trade Agreement (DR-CAFTA), aprobado en 2005 y del que forman parte Estados Unidos, República Dominicana, Costa Rica, El Salvador, Guatemala, Honduras, y Nicaragua.

10 Las cifras comerciales usadas en este epígrafe han sido elaboradas por el autor a partir de datos de Secretaría de Economía de México, año 2005; de la base de datos de comercio World Trade Atlas y de la base de datos de comercio del Instituto para la Integración de América Latina y el Caribe (INTAL) del Banco Interamericano de Desarrollo (BID) DATA INTAL 4.1. 
$188 \%$ del volumen comercial anterior a 1994. Sin embargo, a pesar del gran aumento del comercio de México con el mundo, éste no ha sufrido una diversificación en términos de los lugares de destino.

El comercio total de México con el mundo continua centrándose en la región del TLCAN, representando el lugar de destino y origen del $74 \%$ del intercambio comercial nacional, reduciendo tan solo en un 5\% esta concentración respecto al 79\% registrado en el año 1994.

CUADRO 1. Comercio total de México (en millones de dólares)

\begin{tabular}{|c|c|c|c|c|c|c|}
\hline Año & Mundo & NAFTA & $\begin{array}{c}\text { América } \\
\text { Central }\end{array}$ & $\begin{array}{c}\text { América } \\
\text { Sur }\end{array}$ & UE & Asia \\
\hline $\mathbf{1 9 9 5}$ & 151,994 & 123,839 & 1,043 & 5,540 & 10,184 & 9,776 \\
\hline $\mathbf{1 9 9 6}$ & 185,473 & 151,788 & 1,401 & 7,013 & 11,341 & 12,140 \\
\hline $\mathbf{1 9 9 7}$ & 220,045 & 180,477 & 1,714 & 7,656 & 14,141 & 13,854 \\
\hline $\mathbf{1 9 9 8}$ & 242,833 & 200,129 & 1,909 & 6,895 & 15,958 & 15,124 \\
\hline $\mathbf{1 9 9 9}$ & 278,366 & 231,539 & 1,940 & 6,667 & 18,422 & 17,317 \\
\hline $\mathbf{2 0 0 0}$ & 340,913 & 283,073 & 2,142 & 8,955 & 20,743 & 22,527 \\
\hline $\mathbf{2 0 0 1}$ & 326,839 & 261,850 & 2,132 & 9,685 & 22,075 & 27,656 \\
\hline $\mathbf{2 0 0 2}$ & 329,442 & 257,217 & 2,459 & 10,226 & 22,269 & 34,198 \\
\hline $\mathbf{2 0 0 3}$ & 336,313 & 259,214 & 2,764 & 11,309 & 24,084 & 34,379 \\
\hline $\mathbf{2 0 0 4}$ & 386,504 & 287,161 & 3,299 & 15,509 & 28,291 & 47,564 \\
\hline $\mathbf{2 0 0 5}$ & 435,409 & 312,965 & 4,374 & 19,583 & 34,830 & 58,461 \\
\hline
\end{tabular}

Fuente: Elaboración propia. World Trade Atlas

En cuanto al comercio total entre México y la UE, éste ha disminuido en términos relativos. Lo que en 1993 representaba el $9 \%$ del comercio total mexicano, para el año 2004 representó 7\%. En esta evolución no se puede olvidar el crecimiento del comercio de México con Asia, y especialmente con China.

El fenómeno del desplazamiento del comercio hacia China y algunas otras potencias asiáticas como Taiwan y Corea es una tendencia mundial que ha 
impactado tanto en el comercio mexicano como en el resto de los países y bloques regionales. Tanto el comercio exterior de los Estados Unidos como el de la UE han experimentado un desplazamiento de sus flujos comerciales hacia la zona asiática. En este contexto, la incorporación de China a la OMC, el trato de Nación Más Favorecida (NMF) a las economías emergentes, y el avance que se dio en el programa de las negociaciones aprobadas en la reunión de Doha, son factores que han contribuido a las cambiantes condiciones de competitividad en las que se han desenvuelto los tratados comerciales (Zapata [2004] p. 37) y han derivado en nuevas tendencias en el intercambio comercial de México. De hecho, el comercio total que este país tiene, por ejemplo, con China ha experimentado un incremento del $0,4 \%$ en 1993 al $4 \%$ en 2004.

En general, México ha tenido una tendencia al alza en cuanto al comercio con la mayoría de los países, incluidos los países de Centroamérica y los de América del Sur. Tendencia en la que los intercambios comerciales con la UE, en términos absolutos, no son una excepción. Con la UE el comercio mexicano ha experimentado un aumento del $163 \%$ en el periodo comprendido entre 1993 y 2004.

En definitiva, en el periodo comprendido de 1993 a 2004, en el comercio exterior mexicano resalta el incremento de sus flujos comerciales con China y diversos países de la región asiática, un mayor comercio con los países latinoamericanos, al mismo tiempo que sigue manteniendo fuertes vínculos comerciales con la UE y con Estados Unidos.

En este entorno, para México, la UE sigue siendo el segundo socio más importante, con poco más de 28 mil millones de dólares de comercio total en el 2004. Esta cifra representó el 7\% del total en este año. Sin embargo, es importante destacar que durante el periodo 1993 - 2004, México siempre importó más de lo que exportó a la UE, lo que dio un saldo en balanza comercial siempre deficitario para México. (Cuadro 2)

Respecto del comercio con los distintos países de la UE, en el periodo de 1993 a 2004, la mayor parte del comercio se ha realizado con Alemania. Con este país el comercio total aumentó de un 30\% a un 34\%. En este mismo sentido destaca el caso de Italia, donde los intercambios comerciales pasaron de un $9 \%$ a $12 \%$.

Los países que han disminuido su presencia en el intercambio regional trasatlántico son Francia, quien pasó de tener el 17\% del comercio total a 
tener el 10\% para el 2004, y España quien pasó del 19\% al 18\%. En cuanto a los demás países de la UE, de los 15, los cambios en la composición no fueron significativos. Aun así destaca la presencia de Holanda e Irlanda con el $5 \%$ y $3 \%$ respectivamente en 2004 .

CUADRO 2. Saldo comercial total de México (en millones de dólares)

\begin{tabular}{|l|r|r|r|r|r|c|}
\hline Año & Mundo & NAFTA & $\begin{array}{c}\text { América } \\
\text { Central }\end{array}$ & $\begin{array}{c}\text { América } \\
\text { Sur }\end{array}$ & UE & Asia \\
\hline $\mathbf{1 9 9 3}$ & -13.534 & -1.996 & 478 & -562 & -5.102 & -6.025 \\
\hline $\mathbf{1 9 9 4}$ & -18.529 & -3.273 & 508 & -988 & -6.189 & -7.918 \\
\hline $\mathbf{1 9 9 5}$ & 7.088 & 12.984 & 853 & 1.440 & -3.397 & -5.655 \\
\hline $\mathbf{1 9 9 6}$ & 6.535 & 13.466 & 1.000 & 1.729 & -4.264 & -6.397 \\
\hline $\mathbf{1 9 9 7}$ & 429 & 12.564 & 1.273 & 1.469 & -5.974 & -8.923 \\
\hline $\mathbf{1 9 9 8}$ & -7.914 & 8.972 & 1.435 & 435 & -7.976 & -10.639 \\
\hline $\mathbf{1 9 9 9}$ & -5.584 & 14.357 & 1.258 & -657 & -7.696 & -13.005 \\
\hline $\mathbf{2 0 0 0}$ & -8.003 & 19.189 & 1.240 & -1.316 & -9.586 & -18.113 \\
\hline $\mathbf{2 0 0 1}$ & -9.954 & 25.645 & 1.291 & -1.864 & -11.423 & -23.122 \\
\hline $\mathbf{2 0 0 2}$ & -7.916 & 33.852 & 1.173 & -2.546 & -11.506 & -28.050 \\
\hline $\mathbf{2 0 0 3}$ & -5.603 & 37.854 & 99 & -3.747 & -12.428 & -28.171 \\
\hline $\mathbf{2 0 0 4}$ & -8.103 & 51.659 & 786 & -49.604 & -14.968 & -40.459 \\
\hline
\end{tabular}

Fuente: Elaboración propia. Secretaría de Economía con datos de Banco de México.

En cuanto al saldo comercial que se tuvo con los países miembros de la UE, se tiene un saldo deficitario en la mayoría de los casos durante todo el periodo. Son pocas las excepciones donde el saldo es positivo. Además en todos los casos este indicador muestra una clara tendencia negativa, lo que indica que las importaciones están creciendo más deprisa que las exportaciones para los países miembros. Holanda y Portugal fueron una excepción a esta tendencia general. Con el primero los datos muestran un saldo positivo entre 1998 y 2003 y con el segundo ha habido un saldo positivo entre 1993 y 2003. En ambos casos, en el año 2004 la cifras pasaron a ser deficitarias. De todos los países destaca Alemania con el que se ha mantenido el mayor déficit durante todo el periodo. 
En el año 1995, los principales productos ${ }^{11}$ exportados a la UE eran, como porcentajes de las exportaciones totales, los siguientes: $23,2 \%$ combustibles minerales, aceites minerales y productos derivados; $13,5 \%$ reactores nucleares, calderas, máquinas y aparatos; $8,31 \%$ productos químicos orgánicos; $6,05 \%$ cobre y manufacturas de cobre; $6,03 \%$ fundición, hierro y acero; $5,37 \%$ maquinas, aparatos y material eléctrico y sus partes; $3,73 \%$ materias plásticas y manufacturas de estas materias; $2,99 \%$ vehículos automóviles, tractores, ciclos y demás; $2,57 \%$ minerales, escorias y cenizas; $2,01 \%$ perlas finas y cultivadas y piedras preciosas; y $1,77 \%$ manufacturas de fundición de hierro o de acero. Por lo tanto, 11 capítulos representaban el $75 \%$ de las exportaciones totales.

En este mismo año, la UE exportó los siguientes bienes: 27,1\% reactores nucleares, calderas, máquinas y aparatos; $9,87 \%$ máquinas, aparatos y material eléctrico y sus partes; $8,34 \%$ productos químicos orgánicos; $6,82 \%$ importación de mercancias mediante operaciones espaciales; $6,57 \%$ vehículos automóviles, tractores, ciclos y demás; $4,14 \%$ instrumentos y aparatos de óptica y fotografía; $4,11 \%$ fundición hierro y acero; $2,81 \%$ materias plásticas y manufacturas de éstas; y $2,41 \%$ manufacturas de fundición de hierro o de acero; $2.32 \%$ productos farmaceúticos; y 2,04\% leche y productos lácteos. Estas 11 capítulos representaron el 76\% de las exportaciones totales.

En el año 2004, los principales capítulos de las exportaciones mexicanas hacia la UE eran los siguientes: $28,71 \%$ combustibles minerales, aceites minerales y productos derivados; $12,68 \%$ vehículos automóviles, tractores, ciclos y demás; $9,77 \%$ reactores nucleares, calderas, máquinas y aparatos; 7,08\% máquinas, aparatos y material eléctrico y sus partes; $6,9 \%$ instrumentos y aparatos de óptica y fotografía; $5,7 \%$ productos químicos orgánicos; $4,55 \%$ productos farmaceúticos. Todo esto significó el $75 \%$ de las exportaciones totales concentrado en 7 capítulos.

Para este año, las principales exportaciones de la UE hacia México fueron las siguientes: $21,69 \%$ reactores nucleares, calderas, máquinas y aparatos; $15,18 \%$ máquinas, aparatos y material eléctrico; $11,6 \%$ vehículos automóviles, tractores y ciclos; $6,02 \%$ productos químicos orgánicos; $4,94 \%$ productos farmaceúticos; $4,44 \%$ instrumentos y aparatos de óptica y fotografía; $3,94 \%$ función hierro y

11 Los datos sobre la composición del comercio entre la UE y México han sido elaborados a partir de los datos, según el Sistema Armonizado a dos dígitos, de la base de datos de comercio World Trade Atlas, 2006. Está base está construida con datos de la Secretaría de Economía del gobierno mexicano. 
acero; $3,45 \%$ materias plásticas y manufacturas de éstas; $2,16 \%$ aceites esenciales y resinoides; y 1,89\% manufacturas de fundición de hierro o de acero. En este año 10 capítulos significaron 78\% de las exportaciones totales.

En los datos anteriores, a un nivel muy agregado, podemos observar como México ha sufrido una concentración en su comercio hacia la UE, mientras que este último mantiene un patrón a este nivel muy parecido. Por lo que vemos que la relación comercial con la UE mantiene las asimetrías que ya existían antes del Acuerdo en el camino de un modelo concentrador que se centra en las grandes empresas ligadas al tradicional sector exportador. También podemos observar que los niveles de comercio intraindustrial están situados alrededor del 50\% durante todo el periodo. Esto apunta, como menciona Rozo ([2006] p. 11) de acuerdo a la opinión de algunos representates europeos, "a que el grueso de las exportaciones son realizadas por empresas europeas; es decir, que buena parte de las transacciones de exportación son comercio intra-firma". Todo lo anterior, junto con un crecimiento del comercio total con Estados Unidos dentro del marco del TLCAN ${ }^{12}$, no parece haber permitido generar un marco alternativo para que México pueda diversificar ni productos ni destinos.

México en el TLCUEM se ha convertido en un país principalmente importador de productos provenientes de la UE. Podemos explicar esta tendencia por las oportunidades que ha tenido México de importar nuevos productos provenientes de la UE a partir de la firma del Acuerdo y los costes de transacción elevados que deben asumir los empresarios mexicanos hacia el interior y con la UE. Este déficit se puede explicar, entre otras, por las siguientes razones que dificultan a los empresarios mexicanos la colocación de sus productos en el mercado europeo:

a) una deficiente infraestructura y un pobre uso de la logística y el transporte por parte de las empresas mexicanas, al contrario de lo que ocurre en la UE;

b) la fuerza gravitatoria que el mercado norteamericano ejerce sobre los empresarios mexicanos y que ha hecho que éstos se hayan enfocado demasiado a las ventajas comparativas que tienen con respecto al mercado del norte y que no necesariamente son las que podrían encontrar en el mercado europeo;

12 El comercio total de México con Estados Unidos aumentó de 261,84 millones de dólares en 2001 a 284,47 millones en 2005, según datos de World Trade Atlas, 2006. 
c) un sistema financiero que no facilita el crédito a la pequeña y mediana empresa; y

d) una normativa europea que plantea un sistema de acreditaciones complejo y exigente para los empresarios que quieren exportar a la UE.

Se vuelve una tarea de especial trascendencia para México identificar aquellos productos estratégicos y sensibles, donde este país pueda explotar sus ventajas competitivas con respecto a la UE y así aumentar el volumen de comercio y las exportaciones hacia esta zona. Al mismo tiempo, que se implementan las medidas de acompañamiento que permitan una efectiva y beneficiosa cooperación económica y política. Este modelo de relación entre la UE y México, y con América Latina, marcaría el carácter distintivo en estos acuerdos Norte-Sur, de comercio y cooperación, que legitimarían una estrategia regional en esencia diferente al TLCAN.

\section{La inserción regional de México y su confianza en el regionalismo norte-sur}

Durante toda la década del noventa, México puso toda su confianza en el nuevo modelo nacional e internacional derivado del TLCAN y la firma de acuerdos de libre comercio con países y regiones del mundo, como el TLCUEM. Durante estos años, México ha firmado 1 acuerdo de asociación económica, 11 tratados de libre comercio ${ }^{13}$ y 3 acuerdos de complemantación económica. Por lo tanto, un total de 15 tratados y/o acuerdos con un total de 45 países de 3 continentes. Esta política, o como algunos piensan ausencia de ésta, ha reforzado la posición del mercado norteamericano como el principal socio comercial de este país.

Este proceso de liberalización económica a la sombra de la economía norteamericana pareció favorecer a México. Éste se encontró, de manera espontánea, disfrutando de una serie de ventajas competitivas en el panorama económico mundial. Ante esta situación, los políticos, académicos y empresarios mexicanos naturalizaron la idea de que el Acuerdo de Libre Comercio con Norteamérica y la inserción internacional a través de acuerdos de libre comercio con el mundo era una estrategia de desarrollo en sí misma y donde el Estado no debía aplicar una política deliberada.

13 Incluye al TLCUEM. 
Esta naturalización ha estado fundamentada en la creencia de que el camino del desarrollo nacional está sembrado de este tipo de ventajas que, junto con la IED, irán dando frutos de acuerdo a un ciclo natural. Por lo tanto, se ha pensado que la única estrategia, que la única política, es el conjunto de medidas que permiten la apertura comercial y demás prescripciones del recetario de Washington. El resto, o sea el desarrollo, vendrá dado por "añadidura".

En la edad media, existía una relación irracional entre la divinidad y el hombre medieval que la modernidad rompió a través de las ideas ilustradas. Curiosamente ahora como en el medioevo, y pensando en las ideas de San Agustín, esta modernidad venida del Occidente rico ha creado, a su vez, otra relación irracional entre el liberalismo, como divinidad, y el hombre feliz en el largo plazo, que se caracteriza por una relación dogmática basada en creencias liberales y en su política económica. Se da primacía al mercado, lo trascendente, y se niega otra posibilidad de política económica, por terrenal y mundana. Bajo esta creencia, un Estado, aunque bien gobernado y con sus mejores intenciones, no pasará de ser una imagen de la política verdadera. Dice San Agustín, en este sentido, "incluso cuando ellos hacen lo mejor que ellos pueden, lo mejor está lejos de la justicia verdadera; $y$, a menudo, lo que ellos hacen está lejos de lo mejor" (Losco y Williams [1997] p. 118).

Como hemos mencionado en los párrafos anteriores, México se ha volcado hacia el mercado norteamericano sin preocuparse demasiado por su mercado interno o por diversificar productos o mercados de destino, y los efectos de la entrada en vigor del TLCUEM no parece haber neutralizado esta fuerza gravitaria hacia su mercado del norte, más bien incluso puede estar acentuándola. De hecho, este país ha incrementado notablemente y de manera sostenida el comercio dentro de Norteamérica, especialmente los productos manufacturados, a través de la puesta en marcha de una zona de libre comercio. Sin embargo, el comercio con Canadá y sobre todo con Estados Unidos ha ganado peso relativo respecto del comercio con otros mercados como los europeos, asiáticos o latinoamericanos. Además, siguen manteniendo su carácter de economía exportadora de productos de bajo valor agregado, con escasos niveles de inversión y de desarrollo tecnológico. Mantiene una alta vulnerabilidad a fuertes shocks externos, y un alto nivel de endeudamiento.

En estos tres lustros, México no ha logrado resolver problemas tan esenciales como: disminuir los niveles de pobreza; reducir la polarización 
social y la inseguridad derivada de ésta, que incluso ha crecido; insertar competitivamente a sus empresas en el escenario global y ser un destino cada vez más atractivo para la inversión internacional; generar un modelo de industrialización; y fortalecer sus instituciones. Por lo tanto, podemos decir que, en este sentido, los resultados de la aplicación de los PAE y de la adopción del modelo de integración regional anglonorteamericano están siendo decepcionantes.

En los últimos años, en el panorama económico internacional han emergido nuevos actores, como son los casos de China e India, que se están incorporando a los mercados globales con mucha fuerza y que han empezado a restar terreno a México en el mercado norteamericano y europeo.

México, en este sentido, está pagando el precio por la confianza excesiva depositada en el modelo aplicado desde la década del ochenta. Quizá no debamos culpar sólo al modelo sino también a la actitud de los empresarios y políticos -más de objeto que de sujeto-dentro de este teórico instrumento de desarrollo. La aplicación de los PAE y la participación en el TLCAN y en el TLCUEM, si éste prioriza su vertiente comercial sin medidas efectivas de acompañamiento, no representa todo lo que México debe hacer para una inserción adecuada en el plano internacional. Parafraseando a Baltazar Gracián: hay que usar los medios humanos como si los divinos no existieran, y los divinos como si no existieran los humanos.

De acuerdo con las categorías de Andrew Hurrell ([1995] pp. 50-53), la existencia de un 'hegemón local' insta a los países más débiles, bajo su influencia, a buscar la "integración regional" con éste, esperando que su mera cercanía les retribuya algún beneficio (bandwagoning). Esta actuación regional de los países de menor desarrollo está fundamentada en la creencia de que la cercanía a un gran mercado les ofrece la posibilidad de beneficios materiales. Esta visión podría ser también la que está estimulando la entrada de algunos países latinoamericanos a la lógica de construcción regional que ha marcado el ALCA y los TLC. Esta perspectiva teórica podría explicar el porqué de la incorporación de México al TLCAN, al TLCUEM y la influencia de su modelo regional, dentro del nuevo marco de políticas económicas y comerciales surgido en los años ochenta.

Sin embargo, es importante destacar que esta vía de inserción internacional no siempre tiene por qué ser beneficiosa para los países de menor peso, ya que la integración se mueve más por el deslumbramiento pasivo ante las cifras económico-comerciales que por una estrategia activa y bien diseñada 
de inserción internacional a través de bloques. Desde aquí podríamos también explicar una de las características del regionalismo latinoamericano actual, como es la firma desordenada de acuerdos bilaterales, trilaterales o multilaterales entre los países en desarrollo y con otros países o áreas en el mundo. En algunas ocasiones, los países en desarrollo imaginan que su simple presencia en el máximo número de acuerdos regionales, sin mayor estrategia, les reportará algún beneficio.

\section{El debate en México: retos y perspectivas hacia el desarrollo}

En términos políticos y económicos, en América Latina, el siglo XX ha estado marcado por gobiernos que se han ido sucediendo con una tendencia pendular. Gobiernos democráticos, apoyados por grupos conservadores liberales y muy concentrados en los grupos exportadores, eran apartados del poder por gobiernos de naturaleza autoritaria-y más recientemente autocrática- tras la emergencia de agudas crisis o la pérdida de legitimidad. A su vez, cuando las promesas mesiánicas de estos últimos, -y que casi siempre terminan siendo impuestas de forma autoritaria-, no se cumplían, otra vez los gobiernos, de las características de los mencionados en primer lugar, volvían al poder. El desideratum por alcanzar, unas veces, la sociedad soñada u, otras, la sociedad controlada, permitía que las soluciones y los modelos de los extremos se fueran sucediendo de manera cíclica y reactiva.

Esta tendencia quedaba reflejada en una política económica aplicada como reacción a la que se había utilizado previamente, de acuerdo al modelo anterior. Todo ello, ha terminado suponiendo que estas políticas, normalmente, no han profundizado la acción comenzada por las anteriores, sino que han reaccionado contra la dinámica iniciada por ellas. Sin embargo, el desarrollo económico y social de una nación o de un conjunto de naciones necesita el diseño de una estrategia adecuada, tiempo para aplicarla y medidas en el corto plazo que vayan dejando sentir sus beneficios en la población. Se necesita acción y consenso de todos los actores en el largo plazo, y no reacción periódica: no basta sólo la circunstancia, también se necesita la sustancia. México no ha sido ajeno a esta tendencia pendular y su historia reciente lleva esta marca regional.

México, como el resto de los países del mundo, está obligado a tener un papel económico y político activo en el marco de la globalización. Por lo tanto, México, al igual que los demás países está en el río "que nos lleva" y en 
éste la globalización es su más poderosa corriente. Lo que está marcando, y marcará la diferencia, es cómo éstos están navegando por este río. Sin duda, los que decidan adoptar una posición activa se dotarán de mapa y brújula, instrumental que les permitirá estudiar y analizar la ruta hacia la que se encamina y ver el margen de acción que ésta les deja para actuar.

En nuestra opinión, esta sencilla alegoría diferencia a aquellos países que ven al río y a su corriente como estrategia en sí misma, y se dejan arrastrar con confianza; de aquellos otros que en la misma situación deciden marcar objetivos y estrategias.

Finalmente, el debate sobre la política económica y el modelo de desarrollo ha resurgido en México, y en toda América Latina, tras un periodo de timidez, eufemismos y anatemas en los foros académicos. Baste recordar que durante la última década se eludía el término desarrollo para usar otros como estabilidad, gobernabilidad, etc. Otro elemento importante en este debate es permitir que caigan los mitos respecto a los factores que hacen a la economía mexicana atractiva, como son los costos de mano de obra y la especialización en maquiladoras.

En los primeros años del siglo XXI, México ha podido ver con claridad que las ventajas económicas y competitivas internacionales de las que han disfrutado sus empresas, y que convertían a este país en un lugar atractivo para invertir, no eran inagotables e imperecederas. Sin embargo, la economía mexicana no debe desechar las ventajas competitivas (costo de mano de obra, recursos naturales y humanos, situación geográfica, etc.) que hoy tiene, pero siempre en el horizonte de nuevas ventajas, de nuevas posibilidades, y de nuevos mercados. En resumen, un país creativo, innovador y dinámico dentro de una realidad económica internacional en constante cambio: un pathos propio.

Esta visión optimista mostrada en el párrafo anterior viene, al mismo tiempo, aparejada de ciertas dudas en cuanto a los cambios, los reajustes, la adaptación, la nueva estrategia, y la afinación de los objetivos que se pueden y deben plantear en este país.

Ya hemos comentado cómo el ethos liberal marcó la estrategia de política económica nacional e internacional de México. A lo que añadimos el serio cuestionamiento al que está siendo sometido este periodo. Me pregunto, si las políticas, que se han implementado en los últimos años, realmente están consiguiendo lo que finalmente se persigue: que todos los ciudadanos de 
México vivan mejor. Ahora bien, si esto no está siendo así, entonces habría que preguntarse qué se está haciendo mal; por qué México y sus empresas han perdido competitividad y atractivo para los inversionistas; por qué la economía mexicana podría quedar cada vez más rezagada y desplazada en los mercados internacionales; por qué el TLCUEM no se evalúa actualmente como una alternativa de relación Norte-Sur; y qué implicaciones tiene todo esto de cara al futuro.

Otro elemento que genera dudas está relacionado directamente con el cómo de los reajustes, los cambios y las nuevas propuestas. En este sentido, una gran asignatura pendiente de la política económica mexicana es la fijación de una estrategia frente a la globalización desde los espacios regionales. $\mathrm{Al}$ mismo tiempo, que los actores nacionales, desde la acción, afrontan las reformas que no se han implementado, persisten en el logro de los objetivos fijados y mantienen las estrategias nacionales marcadas.

\section{Conclusiones}

La inserción internacional de México, y de los países de América Latina, ha estado en gran parte signada por los acuerdos de libre comercio y por los procesos de integración hasta el punto que en muchas ocasiones un acuerdo de libre comercio podía ser considerado una estrategia de desarrollo en sí mismo, lo que en la actualidad está siendo fuertemente cuestionado en la región. Como dice David Ibarra ([2005] p. 42):

\footnotetext{
"Los países latinoamericanos liberan los mercados y los desregulan, en la creencia de abrir las puertas a un desarrollo rápido o de ganar el beneplácito del primer mundo. Sin embargo, las esperanzas no concuerdan con las realidades, el ritmo de desarrollo latinoamericano en más de dos décadas (1980-2000) se reduce a la mitad del que alcanzó en los treinta años anteriores y se produce desempleo, marginación $y$ desajustes monstruosos en el mercado de trabajo. De ahí surgen reformulaciones paradignáticas".
}

Si bien el TLCUEM se plantea como el punto más alto de las relaciones entre México y la UE, la relación entre ellos ha pasado por varias etapas hasta llegar a la firma y posterior puesta en marcha de este Acuerdo. Además, en la actualidad, cuando ya han transcurrido seis años desde que entrara en vigor, las evaluaciones que se están realizando sobre el acuerdo son pobres y están centradas en el escaso aprovechamiento para México de éste. Este último punto, nos obliga a pensar en las potencialidades de este marco regional y la consolidación de una relación benéfica para México, y América 
Latina, que legitime y estreche unas relaciones alrededor de un modelo de regionalismo Norte-Sur sensiblemente diferente al que este país ha mantenido con Estados Unidos en los últimos años. En palabras de Rozo, en relación a América Latina y la UE, "trabajar conjuntamente hacia el fortalecimiento de la Cohesión Social o búsqueda de una sociedad en la que ninguno de sus grupos constituyentes se encuentre retrasado en su procesos de desarrollo" (Rozo [2006] p. 1). Por lo tanto, en palabras de la Comisión Europea, "hacer congruente la política de desarrollo de la UE con los propósitos de su propia integración que no es solamente unir países comercialmente sino principalmente construir una sociedad más justa". ${ }^{14}$

Al mismo tiempo que Europa, ante sus nuevos retos, va definiendo su proceso de integración, y sabiendo su poder de referencia en América Latina, debe tener en cuenta su influencia en la definición del modelo de integración latinoamericano hacia dentro y hacia el exterior. La UE en los aspectos que se refieren a la integración política y social es un espejo en el que éstos se miran ante el pobre desempeño para México del TLCAN (sólo hay que acercarse a algunos de los muchos documentos que constantemente hacen mención al proceso de integración europeo para darse cuenta de que esto es así) y del que se espera aparezcan, al menos, algunos reflejos en la relación de la UE con los países de la región latinoamericana. Pero además, este espejo también sirve a la UE para ver sus bases teóricas y paradigma reflejado y darse cuenta de cómo aquéllas son vistas por los latinoamericanos como un referente a tener en cuenta, lo cual ayuda a reforzar la confianza en el suyo propio.

Lo anterior supone reconocer la relación entre la UE y América Latina en términos de interdependencia en vez de dependencia, ya que no sólo lo que ocurre en Europa y el avance del proceso de integración europeo afecta al modelo latinoamericano de integración, sino que también se da la relación inversa. Sin embargo, para que esto sea así tanto la UE como los procesos de integración latinoamericanos necesitan que sus procesos estén inspirados en prácticas coherentes con los objetivos enunciados a alcanzar a través de sus procesos de integración. Al mismo tiempo que los acuerdos de la UE con los países y procesos de integración de la región estén inspirados sobre las mismas bases. Todo esto reforzaría su carácter propio y distintivo a favor de su perfil internacional e independencia como actores en la escena mundial.

14 Ibídem. 
Pensando desde la Unión Europea, el desaparecido Instituto de Relaciones Europeo-Latinoamericanas (IRELA) decía hace unos años acerca de las relaciones birregionales lo siguiente: "La aplicación de una política 'europea' coherente y distintiva en América Latina refuerza automáticamente el perfil internacional de la Unión y le permite afianzarse como actor independiente en la escena mundial" (IRELA [1998] p. 12). Reflexión que igualmente se puede aplicar desde los países y procesos de integración latinoamericanos hacia la Unión Europea.

\section{Bibliografía}

Alburquerque, Francisco (2002), Desarrollo Económico territorial, Sevilla, Instituto de Desarrollo Regional/Universidad de Sevilla.

Caldentey del Pozo, Pedro (2001), El desarrollo económico de Centroamérica en el Marco de la Integración regional, Tegucigalpa (Honduras), BCIE.

CASAs Gragea, Ángel M. (2005), Integración regional y desarrollo en los países andinos, Quito, Universidad Andina Simón Bolívar y ETEA, Corporación Editora Nacional.

CASAs GrageA, Ángel M. (2002), "La economía política internacional de la nueva integración regional de las Américas: El caso de la Comunidad Andina”. Integración \& Comercio (Buenos Aires), 6, 16 (enero-junio): 97-164.

CRUz M. Rodolfo (2003), Las relaciones comerciales multilaterales de México y el Tratado de Libre Comercio con la Unión Europea, México, Instituto de Investigaciones Jurídicas/UNAM.

Devlin, Robert y Estevadeordal, Antoni (2001), ¿Qué hay de Nuevo en el Nuevo Regionalismo de las Américas?, Buenos Aires, INTAL-ITD-STA Documento de Trabajo 7, BID-INTAL, agosto.

FAWCETt, Louise y HurRell, Andrew, Ed. (1995), Regionalism in World Politics. Regional Organization and International Order, Oxford, Oxford University Press.

Forero, Clemencia (1999), "La asociación estratégica desde la perspectiva andina”, en Una asociación estratégica para el siglo XXI, editado por Patricio Leiva. Santiago de Chile, CELARE, pp. 195-233.

GALEANA, Patricia (1999), "Encuentros y desencuentros de una historia compartida”, en Latinoamérica en la conciencia europea. Europa en la conciencia 
latinoamericana, coordinado por Patricia Galeana, México, Archivo General de la Nación/Centro Coordinador y Difusor de Estudios Latinoamericanos, UNAM/Facultad de Filosofía y Letras, UNAM/Consejo Nacional para la Cultura y las Artes/Fondo de Cultura Económica, pp. 92-104.

HuRRell, Andrew (1995), "Regionalism in Theoretical Perspective", en Regionalism in World Politics. Regional Organization and International Order, editado por Louise FAWCETT y Andrew HuRRELl, Oxford, Oxford University Press, pp. 27-55

IBARRA, David (2005), Ensayos sobre economía mexicana, México, Fondo de Cultura Económica.

IRELA (1998), América Latina y Europa más allá del año 2000, Dossier № 65. Madrid, IRELA, septiembre.

Krugman, Paul, "Llorar con Argentina", El País Digital (Madrid), 20 de enero de 2002.

Loscon, J. y L. Williams (1997), Polítical theory, Nueva York, St. Martin`s Press.

Romero, José J. y Rodero, Adolfo (dirs.) (2000), España en la Unión Europea. Más allá del euro, Córdoba, ETEA.

Romero, José J., Caldentey del Pozo, Pedro y Ortega Carpio, Maa Luz (2000), "Siete claves para comprender el proceso de construcción europea". Cuadernos socioeconómicos del BCIE, $\mathrm{n}^{\circ} 26$, Tegucigalpa (Honduras).

Rozo, Carlos A. (2006), La asociación Unión Europea-México: balance y perspectivas, México, mimeo.

Schiff, Maurice y WinTERs, L. Alan (2004), Integración Regional y Desarrollo, Bogotá, Banco Mundial/Alfaomega.

Szymanski, Marcela (2002), "El nuevo acuerdo entre México y la Unión Europea: el primer vínculo de libre comercio entre Europa y el TLC", en Lebrija, Alicia y Stephan Sberro (coord.). México-Unión Europea. El acuerdo de asociación económica, concertación política y cooperación: sus aspectos fundamentales, México, Miguel Ángel Porrúa.

ZAPATA M., Ricardo (2004), "La competitividad de las exportaciones mexicanas frente a Estados Unidos en el Marco del TLCAN" en REZA, Germán DE LA (coord.), Más allá del TLCAN, México, Plaza y Valdés. 